\title{
The Physical Teaching Model of the High Level Sports Team in Our Country's Present Situation and Countermeasure Research
}

\author{
Kaijun Ji, Kaibin Ji, Xueshan Li \\ Competitive Sports, Aviation University of Air Force, China \\ jingyuanshishi@sina.com
}

\begin{abstract}
This essay offers a deeper understanding of the connotation of the combination of sports and education and the situation of existing multiple teaching molds, as well as puts forward a thorough solution of this situation.

Index Terms - cultural education, combination of sports and education, high level sports team
\end{abstract}

\section{Introduction}

We can summarize the connotation of the combination of sports and education to be: under the guidance of scientific development perspective, with the concept of people oriented, aiming at the comprehensive development of sports talents, training the modernization construction qualified sports talents of comprehensive development. On the basis of school education, both sports system and education system exploit their human resources advantages to the fullest. Under the premise of guaranteeing the athletes scientific and systematic training, as well as obeying the principle of education development, they both cooperate and play their own parts, which represents the need for the development of talents both in the field of education and sports, and corresponds the inner needs of the development of talents.

In the course of twenty years practice and exploration, it forms into four models below. Firstly, universities bring in retired professional athletes, that is to say, universities bring in excellent retired athletes from professional leagues. With the gradual improvement of the athletes, this kind of model will come to an end[1]. Secondly, the combination model, namely universities and professional leagues cooperate to create a high level sports team, both of which are in charge of the development of athletes, combining and taking advantage of both sports commission and universities inner resources[2]. Thirdly, universities take in athletes in active service, namely combined with sports system, universities train the professional athletes in active service, and offer them quality - oriented education, taking advantage of their training resources. Most universities will adopt this model at present[3]. Fourthly, the model of coordinated process, that is to say, establish a set of backup talents' physical training system in the process of university - middle school-primary school, which forms an inner individual developing network of the education system, and train high-level as well as high-quality physical backup talents on the basis of education.

\section{The Existing Problems in the Model of China's Combination of Sports and Education}

\section{A. The Simplified Contents of Cultural Education for Athletes}

With the development of times, the requirements for the sports talent have been further improved. The problem of being out of balance in the development of athletes between culture level and physical level in the whole nation system makes people realize that it is not appropriate to only emphasize on the physical achievements and neglect the cultural education for the needs of development in the age of information. As a former Asian champion and famous national champion, Zou Chunlan works in the public bathhouse, and etc. These examples reviewed by media demonstrate that the training of sports talents need cultural education. Nowadays the athletes' cultural education only restricted to basic cultural study. Although they have high competing level, their cultural accomplishment and moral prevailing custom are less than satisfactory. There are always some unjustifiable action and solecism in the competition terrain both at home and abroad. Shalala, the president of international table tennis federation, criticized the Chinese athletes once, and pointed out that the Chinese athletes' professional quality does not match their achievements. He Zhenliang, the Chinese Olympic committee once said, "Education without sports is not complete, sports without education goes wrong. "Recently, China appeals to letting sports return to education on the way of combination of sports and education, developing high level sports talents of comprehensive development.

Education without sports is incomplete education, on the contrary, sports without education is not complete, either. If we separate sports from education, and train " talents" only with the intension of winning medals, this kind of talents will be incomplete, too[4]. This kind point of view is parochial and near-sighted. In the long run, although we bring in the concept of ability development, all other subjects' learning surrounds the contents of competitive sports. We regard the improvement of sports achievements and winning honour for the country as the common pursuit and only value standard, but neglect the development of moral quality and other related qualities. It does not match the economic and cultural highly developed society situation under the system of socialist market economy.

In the aspect of athletes' curriculum teaching, limited curriculum contents are set as the established consequence, 
which are also restricted to the firm master of sports knowledge and technology. The teaching contents are very simplex, and only emphasizes on the teaching of academic subjects. Humanistic concern is still neglected by sports education, which is also the weakest point. As far as sports athletes are concerned, the cultivation of personality should never be ignored. We should train comprehensive talents in an integrating way, who are provided with scientific spirit and humanistic feelings.

In order to solve the lack of the athletes' professional spirit and individual qualities, the teaching contents should aim at the athletes' individual characteristics to fulfill comprehensive education, rather than be restricted to the learning of academic subjects. Let them learn the principle of being a man, respect other people, and have the professional spirit. We should not only train the athletes to be sports talents with high technical level as well as comprehensive quality, but also emphasize on the communication with foreign countries, making the development of China's sports talents rise to a higher rank.

\section{B. The Conflict Problem Between Systematic and Organic Growth of Competitive Sports}

The education system and sports system are two sets of parallel system. They hold different opinions, each act in its own way. Education institutions and daily coordination management mechanism are not scientific, programmed, and standardized. Different universities haven't formed a concrete system on the training, scientific research and the logistics. In the recent 20 years, the combination of sports and education is not ideal. The number of the competitive sports talents that are trained by normal universities is very limited. Although the sports team has changed into some kind of college, students still don't pay enough attention to study. Their abilities can't match their academic achievements. Most students can only make a living in the field of sports, or try their best to find other kinds of jobs which are low in academic level in society. Students' culture quality remains to be improving. The main reason is that the two departments haven't reach an agreement in the recognition of the combination of sports and education. They each perform their own rights and obligations according to their goals and targets. They each has a set of decision-making, managing and operating system for the benefit of their own development. The department of education considers the combination as a great means of improving school's reputation as well as enriching the campus culture. Education hasn't achieved its original status, and there exists laziness in the management. While the sports department regard the combination as the means of solving the problem, which is the import and export in the process of training athletes. The two departments haven't practised the overall layout in the management system, and they haven't carried out the substantial consultation, communication and cooperation. On the operation mechanism, they haven't achieved a unity of the organic coordination, as well as the effective support and protection on the policy and system.

In terms of the athlete issues, there is no regularity in the view of learning and training combined, and no designed conformed to the actual athletes race training mode, either. Competition program and learning process lack proper management. We should put forward a reasonable evaluation system aiming at the athletes. In terms of the coach issues, the generally existing problem is that they lack the experience of participating in the high-standard training and competition, as well as the experience in the guidance of high-level athletes. They also lack communication, and the number of young coaches is relatively small. In terms of funding issues, the educational funding is limited because of different systems. There is no available money for competitive sports in the college education funding, and the money from the sports system is not available for school. Funding is still a dilemma which the combination is faced with in most schools at home. The barrier between the combination of sports and education as well as nation system combination

\section{China Has Formed Two Sets of Relatively Independent System for the High-Level Talents}

In the process of 20 years development for the combination of sports and education. One is the sports department's "nation system", and the other is "sports with education system". The two sets of system haven't found an ideal means of combination so far. Even under the influence of the "nation system", the combination of sports and education is still in the charge of both sports and education systems, and hasn't achieved the real combination.

From the reform and opening - up till now, only during the short thirty years, China's competitive sports has become a power in the world of competitive sports. In the 2008 Olympic Games, China's national team achieved the most gold medals in the world, shortening the distance from other world's competitive sports. Such great consequences can't get away from China's professional training system. In the meanwhile, there arose a lot of problems. Learning and training is always a heated topic in the circle of both sports and education. Owing to the influence of the traditional concept, trainer's qualities and the development of physical science. In order to reach the amount of sports achievements, China mostly adopts the manner of adults' training as the youth's amateur training. Therefore, the combination between learning and training time loses its balance. There is a phenomenon that athletes are high in sports achievements, while low in cultural qualities. Meanwhile, some parents pay more attention to the kids' entry to higher school and employment, and consider it useless for their children to develop their sports achievements.

Although the former national sports commission once made announcements several times that we should enforce the athletes' cultural education, combine sports with education. Because of the influence of the two sets of systems on the talents' training system and the concept. The athletes' cultural education is just a form in most areas. China's high concentrated and administrative leading sports leadership system of the competitive sports still dominates the leading role. So the system issues remain to be settled in the whole direction. 


\section{Perfect China's the Countermeasure of "Combination" Model}

\section{A. Perform the Multi-Cultural Educational Contents on The Basis of Cultural Learning}

Scientific orientation education concept and the establishment of the available operating model is the key to success[5]. The combination's target and concept orientation is the primary issue of solving the development direction. The education system of the teaching concept and contents need correct position. The reason why many parents choose to send their children to Singapore Sports School is that that school emphasizes on cultural academic study in the school mission and concept, whose starting point is all for students. Although the purpose of it resemble China's sports education a lot, both of which are aimed at looking for sports talents. But the Singapore school's concept and mission are very clear. In the school concept, the Singapore school considers " the combination " as the education mode. The quality education should be placed in the first place rather than winning the world championship. The combination of sports and education not just learns how to set up base or make up a team to play games from the foreign countries, but the concept of other foreign countries. The implementation of the combination mode should follow the training law from common people to sports talents and the education law from a natural person to a social person. We should change the thinking manners, as well as the implement people - oriented education idea, and treat it as the starting point and the foothold[6]. The combination of sports and education should not only emphasize the development of the competitive abilities, but also the scientific cultural quality, humanistic quality and the development of moral quality, namely the whole development as the target.

\section{B. Combine Sports with Education, and Build the Scientific Management System}

The combination of sports and education is not a simple one, while it is the transformation from the third level training mode to the combination of sports and education, that is to say, a process of operation and management of the two systems of sports and education combined. We should set up the compliment with the standard target of its own development, compliment with the training plan of its own characteristics, develop the diversified resources configuration, carry out the performance and encourage feedback mechanism and establish expert information platform as well as improve the logistics system.

The combination of sports and education carries out reasonable division of labour, in the meanwhile, adjusts structure, builds administrative mechanism of the combination of sports and education, brings the marketing function into play to the fullest and build the platform reasonably. That basis aims at the management of athletes, coaches, competitive system and security problems. We should set up management regulation as well as administrative detailed rules, strictly carry out the learning requirements of athletes. Although the students' grades are very excellent, and they fail in the academic subjects, the students can't be allowed to take part in the competition. In the management of training and studying time, we can take the measures of America's NCAA as an example, namely make use of the qualification for participating in competition to limit the phenomenon of light weight training study both for coaches and athletes. We should set up detailed rules in the security system. Singapore Sports School employs a lot of famous foreign coaches to strengthen the construction of coach team. We can use the experience of Singapore and hire famous coaches for reference, combined with national conditions. Build a flow mechanism in the use of human resources for hiring coaches between the system of sports and education. On the operation of mechanism, arrange the sports venues to the schools, communities, the club centralized place. It not only make the game regularly for the athletes, but also not delay learning. It needs integrated layout of the two departments, the mutual negotiation as well as set up talent exchange platform.

\section{The Combination of Sports and Education Breakthroughs the System Barriers}

China still belongs to a developing country, and there exists distance compared with the developed countries. Under such condition, if totally negative the sports system, the competitive sports can't get the financial support from China, we will lose a great number of experienced sports management researchers and coaches, as well as the advantages of China's sports system. Liu Peng points out that our attitude is very clear, in terms of the sports system. On the one hand, we should insist, on the other hand, complete.

In the sports system, a number of gold medals and champions came out in a short time in China, making great achievements, but the rights of the athletes' culture education have been deprived mercilessly. The lack of culture has become the fatal injury of the transformation of athletes reemployment, and shaken the base of the China's sports reserve talents' system in a way. We can't totally repudiate various problems of the sports system, and should look at problems from the dialectical and comprehensive view, as well as make a breakthrough of the system barriers. So improvement of the sports system is the effective measures that stimulate from the combination of sports and education to the substantial changes.

The combination of sports and education need development, and improvement of the sports system. The improvement of the sports system should insist on people orientation, the measurement of the scale for reserve sports talents should be all - round development of human being, not just measured by winning gold medals. In the process of insisting and perfection of the sports system, we should consider the basic national conditions, adapt to the demand of the Times, as well as the sustainable development of the sports career as the starting point and the stance. So sports system should fully cooperate with education system from the national sports team to the province and city sports schools, explicit the athletes' culture education management and the concrete working contents, and leaders and coaches play a supplementary role. We should build management registration 
system, incentive system, and cultural level access system. The development of competitive sports should be people oriented, and build college competitive system and network, leading students to explore their own sports abilities as well as learn science and culture education. In the meanwhile, we should carry out the combination of sports and education.

\section{Conclusion}

Widen the development way of thinking of the combination of the sports and education for our national high-level sports team, improve the recognition of the quality development of the athletes, scientific position the combination of sports and education, make full use of the rational allocation and performance optimization, and build the science development and sports career, which is full of far-reaching significance.

Under the demand of In line with international standards and the adaptation of the socialist market economy, we should further insist on and perfect the traditional advantages of the sports system, solidify and develop the competitive sports management system with the characteristics of the developing country, in the meanwhile, establish the combination of sports and education, as well as the management system of government as well as market regulation, realizing the overall consideration, coordinated development, and running the sports according to the law, develop sports through science and education. On the way of the combination of sports and education, carry out the comprehensive structure reform and innovation, which has a significant value in the development of China's sustainable sports career.
We should combine sports with education, make full use of the resources on the level of sports technique, knowledge structure, obey its regular pattern, which act as the leading function both in the sports system and in the scope of citizens. It will promote the process of sports' modernization, science, socialization and popularity, and become the determined factors of the sports career, which are comprehensive , harmonious and sustainable, providing the guarantee for the development of China's sports reserve sport talents, and laying firm foundation of the Chinese sports fully catch up with the world advanced level

\section{References}

[1] Sun Xinrong. The Analysis of the Development Breakthrough of China's Colleges' Combination of Sports and Education Mode. Journal of Nanjing Institute of Physical Education, 2007, 21(2).

[2] Zhejiang Sports Commission Collective Department, Zhejiang Education Commission Sports and Arts Department. The Research of School Extracurricular Activities Sports and Training Mode in the Combination of Sports and Education. Zhejiang Sports Science.1999, 21(5).

[3] Hu Jianbo, Wang Luoqi. The Existing Problems and the Solution Research in the Mode of the Combination of Sports and Education. Journal of Chengdu Sports Institute, 2008, 34(4).

[4] Zhang Yiliang. Make the Life in the Sports Field Wonderful-The Research Road of the Combination of Sports and Education in the Attached High School of Beijing Normal University. The Mass Forum, 2008, 22(2).

[5] Song Xu. The Fetters and Handcuffs as well as the Practical Introspection of the Implement of the Combination of Sports and Education. Guangzhou Sports College Journals, 2008, 28(4).

[6 ]Liu Qi. Talk About the Competitive Sports Talents' Developing Mode under the Background of the Combination of Sports and Education. China'd Talents, 2006, 28(5). 